\title{
Mammographic screening for breast cancer in a resource-restricted environment
}

J P Apffelstaedt, ${ }^{1}$ Dr Med, MMed (Surg), FCS (SA), MBA; L Dalmayer, ${ }^{2}$ MB ChB; K Baatjes, ${ }^{1}$ MB ChB, MMed (Surgery), FCS (SA)

${ }^{1}$ Department of Surgery, Faculty of Medicine and Health Sciences, Stellenbosch University, Tygerberg, Cape Town, South Africa

${ }^{2}$ Department of Radiation Oncology, Faculty of Medicine and Health Sciences, Stellenbosch University, Tygerberg, Cape Town, South Africa

Corresponding author: J P Apffelstaedt (jpa@sun.ac.za)

Background. Mammographic screening is carried out at public sector hospitals as part of clinical practice.

Objective. We report the experience of such screening at Tygerberg Academic Hospital (TBAH), a tertiary referral hospital in the Western Cape Province, South Africa.

Methods. All mammograms performed between 2003 and 2012 at TBAH were analysed regarding patient demographics, clinical data, indication and outcome according to the American College of Radiology Breast Imaging Reporting and Data System (BIRADS). Screening mammography was offered to patients $>40$ years of age and mammograms were read by experienced breast surgeons. Patients with BIRADS 3 and 4 lesions were recalled for short-term follow-up, further imaging or tissue acquisition. Patients with BIRADS 5 lesions were recalled for tissue acquisition. Further imaging, method of tissue acquisition, histology results and use of neo-adjuvant therapy were also recorded. Results. Of 16105 mammograms, 3774 (23.4\%) were carried out for screening purposes. The median age of patients undergoing screening was 54 years. Of 407 women with mammograms that were reported as BIRADS 3 - 5 ( $10.8 \%$ of screening mammograms), 187 (46\% of recalled women) went on to have further imaging only. Tissue was acquired in 175 patients (43\% of recalled women), comprising a biopsy rate of $4.6 \%$ of the total series. The malignancy rate in cases in which tissue acquisition was done was $25 \%$. Forty-three breast cancers were diagnosed (11.4/1 000 examinations). Of the cancers, nine (31\%) were ductal carcinomas in situ. Of 20 invasive cancers, nine (45\%) were $<10 \mathrm{~mm}$ in size. Of the invasive cancers, $40 \%$ were node-positive.

Conclusions. The cancer diagnosis rate indicates a high breast cancer load in an urbanised population.

S Afr Med J 2014;104(4):294-296. DOI:10.7196/SAMJ.7246 


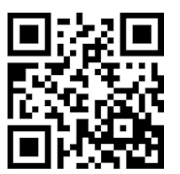

Breast cancer is a growing healthcare burden, especially in the developing world. ${ }^{[1,2]}$ Breast cancer is the most common female cancer in South Africa (SA) and accounts for $20 \%$ of all cancers diagnosed in women in SA. ${ }^{[3]}$ Breast cancer constitutes the largest cancerrelated disease burden in women in the Western Cape (WC) Province, SA. ${ }^{[4]}$ Because limited resources make mammographic breast screening difficult to implement, population screening for breast cancer is not part of WC health policy at present, and will not be within the foreseeable future. ${ }^{[5]}$ Despite this, numerous screening mammograms are undertaken at public sector facilities as part of clinical practice. We report the results of such screening mammograms at an academic teaching hospital in the WC over a period of 9 years.

\section{Methods}

All mammograms performed between 2003 and 2012 at Tygerberg Academic Hospital (TBAH), serving a mostly indigent population in Cape Town, were prospectively entered into MS Access. Data entered included basic patient demographic data such as age and gender, and clinical data such as use and duration of hormone replacement therapy, evidence of previous breast surgery on examination, indication and outcome. Screening mammography was offered to patients referred to the breast clinic who were $\geq 40$ years of age and to younger patients with significant risk factors. Patients who had a palpable mass or personal history of breast cancer were excluded. If a diagnosis of malignancy was made, the tumour stage and detailed histopathological data were recorded, as well as treatment for the malignancy. Screening was classified as 'prevalence' if no prior mammography performed within the past 18 months was available for comparison, or 'incidence' if such imaging was available.

Initially, all mammograms were performed by certified mammographers, using a Senographe Mammography System (GE Healthcare, UK); later, a Giotto 6000 mammography system
(Internazionale Medico Scientifica, Italy) was used. The film used was Mamoray HDR film (Agfa Corporation, USA). Films were developed on an Agfa Classic EOS developer. From 2009, computed radiography was employed and from March 2012, a full-field digital system (Siemens, Germany) was introduced. The views taken were standard craniocaudal and mediolateral oblique; additional views such as 'Cleopatra' views, spot compression and magnification views and 'valley' views were taken, as indicated.

The outcomes of the mammography were classified according to the American College of Radiology Breast Imaging Reporting and Data System (BIRADS) ${ }^{[6]}$ Patients with BIRADS 3 and 4 lesions were recalled for short-term follow-up, further imaging or tissue acquisition. Patients with BIRADS 5 lesions were recalled for tissue acquisition. Further imaging, method of tissue acquisition, histology results and use of neo-adjuvant therapy were also recorded. Patients who failed to present for further work-up, despite contact by mail, were deemed 'defaulters'.

Diagnosed cancers were staged according to the TNM clinical and pathological staging system.

\section{Results}

A total of 16105 mammograms were performed between January 2003 and May 2012, 3774 (23.4\%) for screening purposes. Of these, $48 \%$ were classified as 'prevalence' and $52 \%$ as 'incidence' screening. The median age of women undergoing screening mammography was 54 years; $32 \%$ of the women were 40 - 49 years of age.

Of the screening mammograms, $3367(89.2 \%)$ were reported as BIRADS 1 and 2, $359(9.5 \%)$ as BIRADS 3 and 4 , and $48(1.3 \%)$ as BIRADS 5

Of 407 recalled patients, who represented $10.8 \%$ of all women who had screening mammograms, 187 (46\%) went on to have further imaging that was reported as benign. Tissue was acquired in 175 patients (43\%), constituting a biopsy rate of $4.6 \%$ of the total series. Thirty-four

Table 1. Comparison of the current series with global benchmark programmes

\begin{tabular}{|c|c|c|c|c|}
\hline Criterion & $\mathrm{USA}^{[12]} \%$ & NHS (UK), ${ }^{[13]} \%$ & Breast Screen (Aus), ${ }^{[13,14]} \%$ & Current series, $\%$ \\
\hline \multirow[t]{2}{*}{ Recall rate $^{*}$} & 9.8 & 7.7 (prevalence) & $4-12$ & 10.8 \\
\hline & & 2.8 (incidence) & & \\
\hline Biopsy rate & - & 1.72 & - & 4.6 \\
\hline Malignancy rate of biopsies* & 33.8 & 45.5 & - & 25 \\
\hline \multicolumn{5}{|l|}{ Cancers per age group (years)* } \\
\hline All & 4.7 & - & - & 11.4 \\
\hline $40-49$ & - & 7.8 ( $>45$ years $)$ & 2.4 & - \\
\hline \multirow[t]{2}{*}{$50-69$} & - & - & 12.1 (prevalence) & - \\
\hline & & & 5.7 (incidence) & \\
\hline In situ cancers (of total) & 21.6 & 20.6 & 20.2 & 31 \\
\hline \multicolumn{5}{|l|}{ Invasive cancers, size (mm) } \\
\hline Of total & 78.4 & 79.4 & 79.8 & 69 \\
\hline$<10^{*}$ & 37.2 & - & - & 45 \\
\hline \multirow[t]{2}{*}{$11-20^{*}$} & $31.6^{\dagger}$ & $50.8(<15 \mathrm{~mm})$ & 47 (prevalence) & 20 \\
\hline & & & 63 (incidence ) (<15 mm) & \\
\hline$>20^{*}$ & $21.2^{\dagger}$ & - & - & 30 \\
\hline Node-negative $^{*}$ & 79.8 & 76 & - & 60 \\
\hline Node-positive & 20.2 & 24 & - & 40 \\
\hline
\end{tabular}




\section{RESEARCH}

recalled patients did not present for further work-up, in 4 patients workup was incomplete, 2 patients were found to have lymphoma, and 5 patients died before a definitive diagnosis could be established.

The biopsy rate in cases in which tissues acquisition was done for the whole series was $4.6 \%$; the malignancy rate of tissue acquisition was $25 \%$. Forty-three breast cancers were diagnosed, giving a cancer diagnosis rate of 11.4/1 000 examinations. Of the cancers, nine (31\%) were ductal carcinomas in situ. Of 20 invasive cancers, $45 \%$ (in nine patients) were $<10 \mathrm{~mm}$ in size and $40 \%$ were node-positive. Fourteen patients were excluded from further analysis: 7 failed to return for cancer treatment, 1 had metastatic disease, 2 received neo-adjuvant systemic therapy, and in 4 the work-up was incomplete at the close of the database.

\section{Discussion}

A significant number of mammographic examinations in the public sector are for screening purposes and reflect adherence to good clinical practice. If surveillance mammography after a cancer diagnosis is included, about three-quarters of all mammographies in similar centres to TBAH will be done on asymptomatic women. This calls into question the frequently made distinction between symptomatic and screening mammography. ${ }^{[8]}$ It is our contention that, as soon as a mammography unit is set up, the majority of examinations will be of asymptomatic women. This has important implications for resource allocation in that the required skills and technology must then be in place to address non-palpable lesions.

The screening effort we report here is best described as 'opportunistic' screening. ${ }^{[9]}$ In contrast to organised screening, in opportunistic screening there are no generally agreed performance standards and women are not invited, but present of their own volition for what is sometimes unrelated pathology. ${ }^{[9]}$ Reasons for requesting screening may include a family history of breast cancer which often turns out to be non-significant, mastalgia, referral for a mass in the breast that cannot be verified at physical examination, or simple general concern about breast cancer risk. As such, it is possible to speculate that the breast cancer diagnosis rate would be higher in such a population than in an organised screening programme.

A hallmark of opportunistic screening programmes is their wide variation in quality parameters. ${ }^{[9,10]}$ Despite this, and with the caveat that small numbers do not permit definitive conclusions, the results described here are remarkably similar to those of major international organised screening programmes (Table 1), with the exception of the rate of node-negative invasive cancers. This latter warrants further exploration in a larger programme, especially as the invasive cancers were remarkably similar in size to those in the other programmes quoted. However, the age composition in our series was much younger implying diagnosis of more aggressive cancers. Owing to small numbers, no attempt was made to differentiate between 'prevalence' and 'incidence' screening; this is commonly not done in reports of opportunistic screening efforts.

Recently, much discussion has centred around 'harms' generated by mammographic screening, inter alia psychological distress caused by unnecessary recalls, physical harms caused by unnecessary biopsies and health system harms of resources diverted to screening. These potential 'harms' have led to the controversially restrictive recommendations of the US Preventive Services Task Force for mammographic screening. ${ }^{[11}$ In this series, harms would have fallen within the parameters set by benchmark programmes and are indicative of the rigorous attention to quality at all steps of the diagnostic process.

A much larger proportion (32\%) of screened women in this series than in the benchmark organised screening programmes were in the 40 49-year age group; in this age group, the cancer diagnosis rate is expected to be much lower than in the 50 - 69-year age group. Only $4.3 \%$ of the screened population in the UK National Health Service (NHS) screening programme and $14.3 \%$ in Breast Screen Australia were in this younger age group; in the US programme, where an 'opportunistic' approach was also followed, the proportion was $34.1 \%$. Despite this, the cancer diagnosis rate in our TBAH series is as high as in known high-incidence populations. A reason for this may be the selection of patients from a largely symptomatic service; however, it fits with data from another centre in the same geographical area engaging mostly in screening examinations. ${ }^{[15]}$ The high diagnosis rate calls into question the often quoted figure of a $1 / 28$ lifetime risk of breast cancer in our population. ${ }^{[3]}$ The TBAH screening figures are more in line with those of populations where the lifetime risk is $\sim 1 / 8-1 / 10$ women.

A further noteworthy fact is that this TBAH series was based exclusively on mammography interpretation by surgeons with a special interest in breast health. Despite this, the results compare well with international benchmarks and signal that the desired early diagnosis of breast cancer was achieved. The well-documented shortage of radiologists, specifically breast radiologists, ${ }^{[15]}$ must not prevent the urgent establishment of breast centres to cater for the rapidly rising disease burden of breast cancer in our country while there are trained and experienced surgeons, with a special interest in breast health, available to interpret breast imaging.

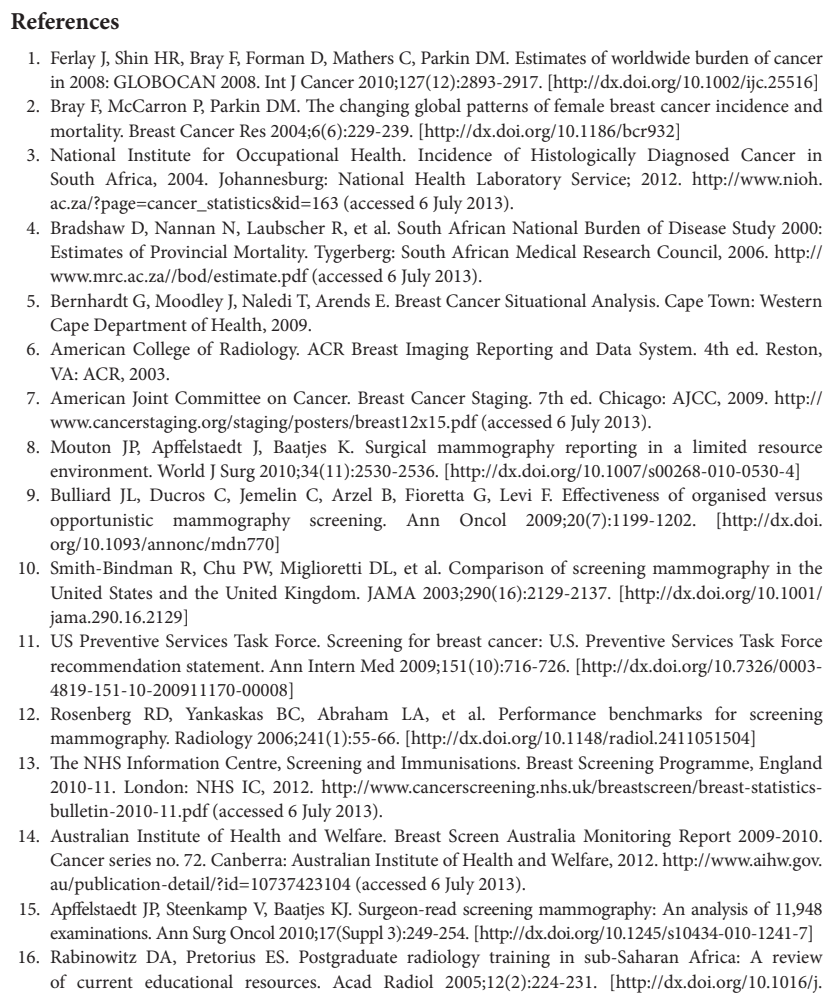

10. Smith-Bindman R, Chu PW, Miglioretti DL, et al. Comparison of screening mammography in the United States and the United Kingdom. JAMA 2003;290(16):2129-2137. [http://dx.doi.org/10.1001/ jama.290.16.2129]

11. US Preventive Services Task Force. Screening for breast cancer: U.S. Preventive Services Task Force recommendation statement. Ann Intern Med 2009;151(10):716-726. [http://dx.doi.org/10.7326/0003 4819-151-10-200911170-00008

12. Rosenberg RD, Yankaskas BC, Abraham LA, et al. Performance benchmarks for screening mammography. Radiology 2006;241(1):55-66. [http://dx.doi.org/10.1148/radiol.2411051504]

13. The NHS Information Centre, Screening and Immunisations. Breast Screening Programme, England 2010-11. London: NHS IC, 2012. http://www.cancerscreening.nhs.uk/breastscreen/breast-statisticsbulletin-2010-11.pdf (accessed 6 July 2013).

14. Australian Institute of Health and Welfare. Breast Screen Australia Monitoring Report 2009-2010. Cancer series no. 72. Canberra: Australian Institute of Health and Welfare, 2012. http://www.aihw.gov. au/publication-detail/:id=10737423104 (accessed 6 July 2013).

15. Apffelstaedt JP, Steenkamp V, Baatjes KJ. Surgeon-read screening mammography: An analysis of 11,948 examinations. Ann Surg Oncol 2010;17(Suppl 3):249-254. [http://dx.doi.org/10.1245/s10434-010-1241-7] 6. Rabinowitz DA, Pretorius ES. Postgraduate radiology training in sub-Saharan Africa: A review of current educational resources. Acad Radiol 2005;12(2):224-231. [http://dx.doi.org/10.1016/. acra.2004.11.014]

Accepted 29 November 2013. 